\title{
A model to analyze the device level performance of thermoelectric generator
}

\author{
Yongjia Wu, Lei Zuo*, Jie Chen, Jackson A. Klein \\ Department of Mechanical Engineering, Virginia Polytechnic Institute and State University, VA, \\ USA, 24060 \\ Corresponding author: Lei Zuo \\ Email: $\quad$ leizuo@vt.edu
}

\begin{abstract}
The thermoelectric generator (TEG) is a distinctive solid-state heat engine with great potential in various scale energy harvesting. Device-level heat transfer coupled with energy conversion makes the accurate analysis of the system very complicate. In this paper, the thermodynamic analysis in a TEG module is carried out to study the influence of the contact layer resistance, Thompson Effect, Joule heat, and thermo-pellet gap heat leakage on the performance of the TEG. All expressions of power output, current, matching load resistant factor, and efficiency of the device are derived and compared with a commercial module. The equations for the simplified model are also given concisely in order to give a full picture of TEG modeling. The research can evaluate the combined influence of all the factors and redress some derivations in the existing models.
\end{abstract}

Keywords

Thermoelectric generator; Power generation; Thompson Effect; Contact resistance; Heat leakage

\section{Nomenclature}

\begin{tabular}{|c|c|}
\hline$\Delta T, T_{h}, T_{c}$ & $\begin{array}{l}\text { Temperature difference, hot and cold end temperatures of } \\
\text { thermo-pellets }(\mathrm{K})\end{array}$ \\
\hline$T, T_{H}, T_{C}$ & $\begin{array}{l}\text { Temperature, hot and cold temperatures of TEG module } \\
(\mathrm{K})\end{array}$ \\
\hline$Z, Z_{P N}, Z_{P N}^{c}$ & Thermoelectric figure-of-merit of material \\
\hline$\eta, \eta_{c}, \gamma$ & Efficiency, Carnot efficiency, and reduced efficiency \\
\hline$\lambda_{P}, \lambda_{N}, \lambda_{g}, \lambda_{C H}, \lambda_{C C}$ & $\begin{array}{l}\text { Thermal conductivities of } \mathrm{P}, \mathrm{N} \text {-type thermoelectric } \\
\text { materials, filling gas, hot end ceramic cover and cold end } \\
\text { ceramic covers }(\mathrm{W} /(\mathrm{m} \cdot \mathrm{K}))\end{array}$ \\
\hline$A_{P}, A_{N}, A_{g}, A_{C H}, A_{C C}$ & $\begin{array}{l}\text { Cross-section areas of } \mathrm{P}, \mathrm{N} \text {-type thermo-pellets, filling gas, } \\
\text { hot and cold end ceramic covers }\left(\mathrm{m}^{2}\right)\end{array}$ \\
\hline$L_{P}, L_{N}$ & Leg length of P, N-type thermo-pellets (m) \\
\hline
\end{tabular}




$$
\begin{aligned}
& K_{P}, K_{N}, K_{P N}, K_{C H}, K_{C C} \\
& \varepsilon \\
& \rho_{P}, \rho_{N} \\
& R_{P}, R_{N}, R_{P N} \\
& \tau_{P}, \tau_{N}, \tau_{P N} \\
& q, q_{h}, q_{c} \\
& \alpha, \alpha_{P}, \alpha_{N}, \alpha_{P N}
\end{aligned}
$$$$
\alpha_{P}^{h}, \alpha_{P}^{c}, \alpha_{N}^{h}, \alpha_{N}^{c}, \alpha_{P N}^{h}, \alpha_{P N}^{c}
$$$$
R_{c h}, R_{c c}, R_{c}
$$$$
R_{G}, R_{L}
$$$$
P
$$$$
\text { I }
$$$$
u, v, w, \theta
$$$$
K_{P}^{*}, K_{N}^{*}, R_{P}^{*}, R_{N}^{*}
$$$$
s, r_{c}, r_{c c}, r_{c h}
$$$$
K_{H}, K_{C}, K_{C H}, K_{C C}, K_{C C H}, K_{C C C}
$$$$
K_{S H}, K_{S C}
$$$$
f_{h}, f_{c}
$$

Thermal conductance of $\mathrm{P}, \mathrm{N}$-type thermo-pellets, thermal conductance of a thermocouple, thermal conductance of hot and cold end ceramic covers $\left(\mathrm{W} / \mathrm{m}^{2} \cdot \mathrm{K}\right)$

Emissivity of the ceramic plate surface

Stefan-Boltzmann constant $\left(\mathrm{W} \cdot \mathrm{m}^{-2} \cdot \mathrm{K}^{-4}\right)$

Electrical resistivity of $\mathrm{P}, \mathrm{N}$-type thermoelectric materials $(\Omega \cdot \mathrm{m})$

Electrical resistance of $\mathrm{P}, \mathrm{N}$-type thermoelectric materials, electrical resistance of a thermocouple $(\Omega)$

Thompson coefficients of P, N-type thermoelectric materials, Thompson coefficient difference of $\mathrm{P}, \mathrm{N}$-type thermoelectric materials $(\mathrm{V} / \mathrm{K})$

Total heat flow, hot end heat absorption and cold end heat absorption (W)

Seebeck coefficient, Seebeck coefficients of P, N-type thermoelectric materials, Seebeck coefficient difference of $\mathrm{P}, \mathrm{N}$-type thermoelectric materials $(\mathrm{V} / \mathrm{K})$

Seebeck coefficients of $\mathrm{P}, \mathrm{N}$-type thermoelectric materials at the hot and cold ends, Seebeck coefficient difference of $\mathrm{P}, \mathrm{N}$-type thermoelectric materials at hot and cold ends $(\mathrm{V} / \mathrm{K})$

Electrical contact resistance at the hot and cold ends of thermo-pellets, total electrical contact resistance $(\Omega)$

Internal resistance of TEG, load electrical resistance $(\Omega)$

Power output (W)

Electrical current (A)

Non-dimensional factors

Reduced thermal conductance $\left(\mathrm{W} / \mathrm{m}^{2} \cdot \mathrm{K}\right)$, reduced electrical resistance $(\Omega)$

Electrical load resistance factor, total contact layer electrical resistance factor, hot and cold end electrical contact resistance factors

Thermal conductance of the hot and cold end ceramic covers, hot end and cold end ceramic cover thermal conductance, hot and cold end contact layer thermal conductance $\left(\mathrm{W} / \mathrm{m}^{2} \cdot \mathrm{K}\right)$

Hot and cold end heat sink/exchanger thermal conductance $\left(\mathrm{W} / \mathrm{m}^{2} \cdot \mathrm{K}\right)$

Hot and cold end TEG module thermal conductance factor 


\section{Introduction}

The thermoelectric generator (TEG) is a solid-state device which converts thermal energy into electricity based on Seebeck Effect without any moving parts. When there exists a temperature difference between the hot and cold ends of TE material, the charge carriers (electrons, $\mathrm{e}^{-}$, in $\mathrm{n}$-type materials and holes, $\mathrm{h}^{+}$, in p-type materials) at the hot side move to the cold side, producing an electrostatic potential. The Seebeck Effect was first discovered in metal in 1821[1], when Thomas Johann Seebeck, a German scientist, found that a compass needle defected when the joint of two conductors was heated. But the effect did not arouse much attention because the Seebeck coefficient of metal was very small (typically less than $10 \mu \mathrm{V} / \mathrm{K}$ ). With the discovery of semiconductors and its alloys with high Seebeck in 1950s[2], the new potential of thermoelectric technology refocused people's attention. The classical thermoelectric materials[3] including $\mathrm{Bi}_{2} \mathrm{Te}_{3}$ and its alloys with $\mathrm{Bi}_{2} \mathrm{Se}_{3} / \mathrm{Sb}_{2} \mathrm{Te}_{3}$ working in low temperature ranges, $\mathrm{PbTe}$ and its alloys with $\mathrm{PbSe} / \mathrm{SnTe}$ in medium temperature ranges, and SiGe alloys in high temperature ranges, all have Seebeck coefficients of more than $200 \mu \mathrm{V} / \mathrm{K}$, making it possible to develop generators or coolers based on thermoelectric materials. The device has many advantages over other conventional energy harvesting technologies, including quietness, small size, cleanliness, high energy density, long lifecycle, and simplicity. TEGs are currently widely used in applications ranging from power generators in space missions[4], common thermocouple sensors[5], small energy harvesters[6] for self-powered sensors[7], to automobile exhaust energy harvesting[8]. Small TEGs can be integrated directly onto key industrial components, including pipes, pump housings, heat exchangers, reactor vessels, boiler bodies, distillation columns, shielding structures, and many other components, acting as reliable energy sources to power monitoring sensors, control circuits, and communications equipment.

The technology has receiving intensive attention in recent years as the efficiency of the devices has been greatly increased thanks to the impressive progress in the nano materials [9] and thermal design technology since the 1990s [10]. According to new research, the highest ZT reported was 3.5 in Bi-doped n-type $\mathrm{PbSeTe} / \mathrm{PbTe}$ quantum-dot super-lattice by Harman et al.[11], and the corresponding energy conversion efficiency is expected to reach $20 \%$. Another work done by Venkatasubramania et al.[12] reported a thin-film $\mathrm{Bi}_{2} \mathrm{Te}_{3} / \mathrm{Sb}_{2} \mathrm{Te}_{3}$ super-lattice device with a ZT value as high as 2.4. All these progresses significantly extend the potential application range of the devices. There were no evidence showed that there were ZT limitation for thermoelectric material. Mahan and Sofo[13] thought that $\mathrm{ZT}=14$ were achievable in rare-earth compounds. An inconvenient truth about thermoelectric is that, until now, the conversion efficiency of TEG is still far less than mechanical thermal engines[14]. For the moment, the practical and economic applications of this technology are still limited in relatively small scale, decentralized energy harvesting.

With the ever-rising demand for energy and urgency for $\mathrm{CO}_{2}$ emission reduction, thermoelectric technology, being clean and renewable, is a potential candidate for waste-heat harvesting as well as for small scale power generation from various heat sources[15-17]. For example, more than two thirds of the heat produced in automobile is discharged into the surrounding. If ten percent of the waste energy is recovered by TEG modules, the total amount of energy saved will be huge. Moreover, nuclear plants accidents (Chernobyl, Three Mile Island, and Fukushima Daiichi) have cast a shadow on the history and future of nuclear power. The security problem in severe situation monitoring is the first consideration in industrial community. 
Self-powered sensing system aroused much interest these years. TEG is one of the most promising alternatives for the conventional cable power supply system for the sensing and monitoring in nuclear accidents[18].

There are many mathematical models built to analyze the performance of TEG models[16, 19-21]. In most cases, people neglected the contact layer thermal and electrical resistance, Thompson Effect, and heat leakage to simplify their models[15]. However, with the development of MEMS, more subtle TEGs/TECs are requiring for small scale fabrication[12]. And more accurate, resilient device level analysis is needed to evaluate their performance. The conceptual design and optimization of TEG are still main concerns in TEG research. An accurate analysis relies on a more sophisticated mathematical model. The performance of the TEG affected by Fourier's heat conductivity, Peltier Effect and Joule heat generation rate has been analyzed by many research works[20-23]. The influence of the Thomson effect on the performance of a thermoelectric generator was also studied by some articles[23], though in most cases, the Thompson Effect will has relatively small impact on the whole performance of TEG. In real situation, heat sink and heat exchanger are fixed at the cold and hot end of TEG to maximum its efficiency and power output. Application of thermoelectric energy conversion from thermal to electricity requires careful device level analysis[21]. In addition, in the actual TEG module, there are many layers, such as a diffusion barrier between thermos-pellets and interconnectors, air or thermal insulation materials filling the gap between $\mathrm{P}$, N-type thermos-pellets, and thermal grease layer between different components. Only when all these factors are taken into consideration, will we give a precise evaluation of the performance of a TEG module.

The most widely used thermodynamic model to evaluate the performance of TEG module is the ideal one-dimensional TEG model given in many books, such as[24]. This model assumes that the contact layer is ideal with no resistance; there is no heat leakage and no material properties change in the module. The model is coarse and, in most cases, will overestimate the performance of the device. Subsequently, researchers develop more accurate models to make more precise description of thermoelectric device. Min and Rowe[25] investigated the effect of thermo-pellets length on the module's coefficient of performance (COP) and heat pumping capacity. The results showed that the performance of TEG was largely deteriorated by the thermal/electrical contact resistances, particularly when the thermo-pellet length was small. As the Thompson Effect was secondary in thermoelectric modules, it can be neglected in most cases without much derivation. However, more accurate analysis found that Thompson Effect can significantly change the temperature profile in the thermos-pellets and consequently influence their performance. Freunek et al.[23] put forward a model including the Thomson Effect, the Peltier heat, a parameterization of the Joule heat, as well as all thermal and electrical resistances. The model obtained the temperature difference between the hot and cold ends of the thermos-pellets, but the mathematical expressions were too complicated. They only gave power output and efficiency expressions with Thompson coefficients neglected. Huang et al.[26] analyzed the influence of the Thomson Effect on the maximum attainable temperature difference in the thermoelectric cooler (TEC). They suggested that COP can be improved by taking advantage of the Thomson effect in some cases. Chen et al.[20] conducted a non-equilibrium thermodynamic study on a couple of thermos-pellets to assess the influence of Thompson Effect. In their research, a constant Thompson coefficient was introduced to avoid nonlinear thermodynamic equations. However, the model did not give the maximum efficiency and power output of the device, and the research was also limited to the 
thermos-pellet level.

The device level efficiency of the TEG is always much lower than the ideal situation because the temperature drop in the heat sink and encapsulation is significant. Hsiao[15] developed a mathematic model of thermoelectric module with applications on waste heat recovery from automobile engine. With a careful thermal network analysis, they obtained the effective temperature difference between the hot and cold junctions of TEG against the engine speed, consequently, got the power, current, and efficiency. Heat leakage is another important factor for precise TEG modeling. The heat can escape into the surrounding without passing the thermo-pellets. In Siddig's doctoral dissertation[21], a unified model was established in the sense that it accounted for the effect of all the parameters that contribute to the performance of the thermoelectric module, some of which were ignored by the available design models. In the model, radiation heat loss and heat flow through the gas gap, contact layer thermal resistance, and ceramic plate resistance were all considered.

In the past decades, the world has witnessed a quick development in thin film TEG fabrication by employing the electrochemical MEMS[27], CVD[28, 29], spray[30], and sputtering[31] methods. The thin film thermoelectric module is compact and flexible, having very short thermal response time and very high energy density[29]. The small energy harvester can be integrated into various heat source surfaces to supply energy for low power applications such as wireless sensor networks[32], intelligent homes, condition monitoring, and mobile devices[33, 34]. In most devices, the thermoelements are arranged in a direction parallel to the deposition plane[35]. While losing some efficiency due to the thermal shorting caused by the substrate material, this layout will gain more profits from higher leg density and larger length of thermoelements. The typical length of a commercial TEG ranges from $1 \mathrm{~mm}$ to $5 \mathrm{~mm}$. The size of thin film TEG can be reduced to less than $100 \mu \mathrm{m}$ [34-36]. When the device reduced to micrometer scale, the factors that notably influence the performance of the device should be very different from the bulk thermoelectric module. There is an urgent need to develop a more accurate mathematical model for small scale thermoelectric module thermodynamic analysis.

As mentioned above, TEG energy harvesting technology has great potential in the future, though it still faces some challenges now. As the temperature gradient becomes larger, the possibility of thermal contact failure increases and the Thomson Effect becomes much more significant. What's more, with the TEGs/TECs reduction to micrometer scales, the contact resistance can no longer be neglected. Most of the models available now missed some factors which might became significant in these situations. This paper build a more accurate mathematical model to analyze the performance of the TEG module, including consideration of Fourier's heat conductivity, Peltier Effect, Thompson Effect, contact layer electrical and thermal resistance, and Joule heat generation rate. In addition, the proposed model is validated by the experiment data of a commercial TEG module provided by a company. A further step is taken to derive the device level performance parameters by introducing thermal resistance of heat sinks at the hot and cold ends. The model will minimum the possible errors introduced by some unnecessary simplifications and provide theoretical basis for the optimization of the design of TEG devices.

\section{Thermodynamic analysis of thermos-pellets}

A thermoelectric generator is constructed by $\mathrm{P}, \mathrm{N}$-type pellets connected electrically in series 
and thermally in parallel (Figure 1(a)). The thermal-electrical conversion efficiency of an ideal one-dimensional TEG can be described as

$$
\phi_{\max }=\eta_{c} \gamma=\frac{T_{\mathrm{h}}-T_{\mathrm{c}}}{T_{\mathrm{h}}} \frac{\sqrt{1+Z T}-1}{\sqrt{1+Z T}+T_{\mathrm{c}} / T_{\mathrm{h}}}
$$

The performance of TEG is dependent upon the thermoelectric properties of the material, and the hot and cold junction temperatures. In a real situation (Figure 1 (b)), the performance of a TEG can be much more complicated when the electrical and thermal resistance, Thompson effect, heat leakage and temperature drop in the heat sinks at the hot and cold end are taken into consideration. In the conventional method, we simplify the heat term by assuming that all the contact regions are ideal and the properties of the TE materials temperature independent. The contribution of the Thomson Effect is also neglected in many research papers[15], on the assumption that it is relatively small, which is true only when the device is operated at relatively low temperature difference and the Seebeck coefficients of TE materials show little variations. Here we establish our mathematical model based on thermodynamic analysis. Some dimensionless parameters are introduced to make the model adaptable for different TEG device modeling.

The cross-section areas of $\mathrm{P}, \mathrm{N}$-type thermos-pellets are set to be $A_{P}$ and $A_{N}$. The lengths of $\mathrm{P}$, N-type thermos-pellets are $L_{P}$ and $L_{N}$. Assuming the average thermal conductivity of P, N-type TE materials are $\bar{\lambda}_{P}$ and $\bar{\lambda}_{N}$, the thermal conductance of $\mathrm{P}, \mathrm{N}$-type thermo-pins are given by

$K_{P}=\frac{\bar{\lambda}_{P} A_{P}}{L_{P}}, \quad K_{N}=\frac{\bar{\lambda}_{N} A_{N}}{L_{N}}$

Similarly, the thermal conductance of ceramic cover in the hot and cold end

$K_{C H}=\frac{\lambda_{C H} A_{C H}}{L_{C H}}, \quad K_{C C}=\frac{\lambda_{C C} A_{C C}}{L_{C C}}$

The P, N-type thermo-pellets gap across-section area is set as $A_{g}$. Then the cross-section area of a TEG can be described as

$A_{C H}=A_{C C}=A_{P}+A_{N}+A_{g}$

Assuming the average electrical resistivity of $\mathrm{P}, \mathrm{N}$-type TE materials are $\bar{\rho}_{P}$ and $\bar{\rho}_{N}$, the electrical resistance of $\mathrm{P}, \mathrm{N}$-type thermo-pellets are

$R_{P}=\frac{\bar{\rho}_{P} L_{P}}{A_{P}}, \quad R_{N}=\frac{\bar{\rho}_{N} L_{N}}{A_{N}}$

The Thompson Effect is a function of temperature and has the Kelvin relationship with Seebeck coefficient. In some studies, the Thompson coefficient is approximated as a polynomial function of temperature[37]. Here we assume it is a constant to simplify the calculation. The assumption is of good precision when the temperature drop in the material is not large and the Seebeck coefficient of the thermoelectric material varies monotonically with temperature $(\tau=T \partial \alpha / \partial T)$.

The thermodynamic control equations in one-dimensional thermo-pellets are

$\mathrm{N}: \frac{d^{2} T}{d x^{2}}+\frac{I^{2} \bar{\rho}_{N}}{\bar{\lambda}_{N} A_{N}{ }^{2}}-\frac{I \bar{\tau}_{N}}{\bar{\lambda}_{N} A_{N}} \frac{d T}{d x}=0$ 
P: $\frac{d^{2} T}{d x^{2}}+\frac{I^{2} \bar{\rho}_{P}}{\bar{\lambda}_{P} A_{P}{ }^{2}}+\frac{I \bar{\tau}_{P}}{\bar{\lambda}_{P} A_{P}} \frac{d T}{d x}=0$

Solving the equations above, the temperature profiles in the $\mathrm{P}$, N-type thermos-pellets are described as follows

$\mathrm{N}: T(x)=\frac{T_{\mathrm{h}}-T_{c}-\frac{I \bar{\rho}_{N}}{\bar{\tau}_{N} A_{N}} L_{N}}{e^{\frac{\bar{\tau}_{N} L_{N}}{\bar{\lambda}_{N} A_{N}}}-1}\left(e^{\frac{\bar{\tau}_{N} I}{\lambda_{N} A_{N}} x}-1\right)+\left(\frac{I \bar{\rho}_{N}}{\bar{\tau}_{N} A_{N}}\right) x+T_{c}$

$\mathrm{P}: \quad T(x)=\frac{T_{\mathrm{h}}-T_{c}+\frac{I \bar{\rho}_{P}}{\overline{\bar{\tau}}_{P} A_{P}} L_{N}}{e^{\frac{\bar{\tau}_{P} I L_{N}}{\bar{\lambda}_{P} A_{P}}}-1}\left(e^{\frac{\overline{\bar{\tau}}_{P} I}{\overline{\bar{p}}_{P} A_{P}} x}-1\right)-\left(\frac{I \bar{\rho}_{P}}{\bar{\tau}_{P} A_{P}}\right) x+T_{c}$

where $\tau(T d \alpha / d T)$ is the Thompson coefficient, $I$ is the electrical current, $T_{h}$ and $T_{h}$ are the hot and cold end temperature of the thermos-pellets, respectively.

Heat flux in the cross-section can be calculated by

$q=I \alpha T+\lambda A \frac{d T}{d x}$

Energy balances are maintained at the hot and cold ends of the devices. The heat flux in the hot/cold-end respectively is given by

$$
\begin{aligned}
& q_{h}=q_{N h}+q_{P h}=\left(\alpha_{P}^{h}-\alpha_{N}^{\mathrm{h}}\right) T_{h} I+\left.\bar{\lambda}_{N} A_{N} \frac{d T}{d x}\right|_{\mathrm{x}=L_{N}}+\left.\bar{\lambda}_{P} A_{P} \frac{d T}{d x}\right|_{\mathrm{x}=L_{P}} \\
& q_{\mathrm{c}}=q_{N c}+q_{P c}=\left(\alpha_{P}^{c}-\alpha_{N}^{\mathrm{c}}\right) T_{c} I+\left.\bar{\lambda}_{N} A_{N} \frac{d T}{d x}\right|_{\mathrm{x}=0}+\left.\bar{\lambda}_{P} A_{P} \frac{d T}{d x}\right|_{\mathrm{x}=0}
\end{aligned}
$$

where the Seebeck coefficients of P, N-type TE materials at the hot and cold end are $\alpha_{P}^{h}, \alpha_{P}^{c}$, $\alpha_{N}^{h}, \alpha_{N}^{c}$, respectively. Substituting Eq. (8)-(9) into Eq. (11)-(12) yields

$$
\begin{aligned}
& q_{h}=\left(\alpha_{P}^{h}-\alpha_{N}^{\mathrm{h}}\right) T_{h} I+\left(K_{P}^{*}+K_{N}^{*}\right)\left(T_{h}-T_{c}\right)+I^{2}\left[R_{P}^{*}+R_{N}^{*}-\left(R_{P}+R_{N}\right)\right]- \\
& \left(\tau_{P}-\tau_{N}\right)\left(T_{h}-T_{c}\right) I-I^{2}\left(R_{c h, P}+R_{c h, N}\right) \\
& q_{c}=\left(\alpha_{P}^{c}-\alpha_{N}^{\mathrm{c}}\right) T_{c} I+\left(K_{P}^{*}+K_{N}^{*}\right)\left(T_{h}-T_{c}\right)+I^{2}\left(R_{P}^{*}+R_{N}^{*}\right)+I^{2}\left(R_{c h, P}+R_{c h, N}\right)
\end{aligned}
$$

where

$$
\begin{aligned}
& K_{P}^{*}=\frac{\tau_{P} I}{\mathrm{e}^{\frac{\tau_{P} I L_{P}}{\lambda_{P} A_{P}}}-1}, K_{N}^{*}=\frac{\tau_{N} I}{1-\mathrm{e}^{-\frac{\tau_{N} I L_{N}}{\lambda_{N} A_{N}}}} \\
& R_{P}^{*}=R_{P}\left[\frac{\lambda_{p} A_{p}}{\tau_{p} I L_{P}}-\frac{1}{\mathrm{e}^{\frac{\tau_{P} I L_{P}}{\lambda_{P} A_{P}}}-1}\right], R_{N}^{*}=R_{N}\left[\frac{\lambda_{N} A_{N}}{\tau_{N} I L_{N}}-\frac{1}{1-\mathrm{e}^{-\frac{\tau_{N} I L_{N}}{\lambda_{N} A_{N}}}}\right]
\end{aligned}
$$

In Eq. (13)-(14), the first term describes heat absorbed by Peltier Effect, the second term specifies the Fourier conductivity, the third term explains the Joule heat, the fourth term shows the Thompson heat and the last term is the contact layer Joule heat.

The electrical current passing the thermocouple can be expressed as

$$
I=\frac{T_{\mathrm{h}}\left(\alpha_{P}^{h}-\alpha_{N}^{h}\right)-T_{\mathrm{c}}\left(\alpha_{P}^{c}-\alpha_{N}^{c}\right)-\left(\tau_{P}-\tau_{N}\right)\left(T_{h}-T_{c}\right)}{R_{P}+R_{N}+R_{c h, P}+R_{c h, N}+R_{c c, P}+R_{c c, N}+R_{L}}
$$


Defining dimensionless parameters as follows

$$
\begin{aligned}
& \alpha_{P N}^{h}=\alpha_{P}^{\mathrm{h}}-\alpha_{N}^{\mathrm{h}}, \\
& \alpha_{P N}^{c}=\alpha_{P}^{\mathrm{c}}-\alpha_{N}^{\mathrm{c}}, \\
& R_{P N}=R_{P}+R_{N}, \\
& R_{c h}=R_{c h, P}+R_{c h, N}=\left(r_{c h, P}+r_{c h, N}\right) R_{P N}, \\
& R_{c c}=R_{c c, P}+R_{c c, N}=\left(r_{c c, P}+r_{c c, N}\right) R_{P N}, \\
& R_{c}=R_{c c}+R_{c h}=\left(r_{c c}+r_{c h}\right) R_{P N}, \\
& R_{G}=R_{P N}+R_{c}=\left(1+r_{c}\right) R_{P N}, \\
& R_{L}=s R_{P N}, \\
& \Delta T=T_{h}-T_{c}, \\
& K_{P N}=K_{P}+K_{N}, \\
& \tau_{P N}=\tau_{P}-\tau_{N},
\end{aligned}
$$

The expression for $I$ and $q_{h}$ can be simplified to

$$
\begin{aligned}
& I=\frac{T_{\mathrm{h}} \alpha_{P N}^{h}-T_{\mathrm{c}} \alpha_{P N}^{c}-\tau_{P N} \Delta T}{R_{G}+R_{L}} \\
& q_{h}=\alpha_{P N}^{h} T_{h} I+\left(K_{P}^{*}+K_{N}^{*}\right) \Delta T+I^{2}\left(R_{P}^{*}+R_{N}^{*}-R_{P N}\right)-\tau_{P N} \Delta T I-I^{2} R_{c h}
\end{aligned}
$$

The power output of the model is given by

$$
P=q_{h}-q_{c}=I^{2} R_{L}=\left(T_{\mathrm{h}} \alpha_{P N}^{h}-T_{\mathrm{c}} \alpha_{P N}^{c}\right) I-\left(\tau_{P}-\tau_{N}\right) \Delta T I-I^{2} R_{P N}-I^{2} R_{\mathrm{c}}
$$

The efficiency of only one couple of $\mathrm{P}, \mathrm{N}$-type thermos-pellets is as fellows,

$$
\eta=\frac{P}{q_{h}}=\frac{\left(T_{\mathrm{h}} \alpha_{P N}^{h}-T_{\mathrm{c}} \alpha_{P N}^{c}\right) I-\left(\tau_{P}-\tau_{N}\right) \Delta T I-I^{2} R_{P N}-I^{2} R_{\mathrm{c}}}{\alpha_{P N}^{h} T_{h} I+\left(K_{P}^{*}+K_{N}^{*}\right) \Delta T+I^{2}\left(R_{P}^{*}+R_{N}^{*}-R_{P N}\right)-\left(\tau_{P}-\tau_{N}\right) \Delta T I-I^{2} R_{c h}}
$$

Substituting the dimensionless parameters into Eq. (21) yields,

$$
\eta=\frac{P}{q_{h}}=\frac{\left(T_{\mathrm{h}} \alpha_{P N}^{h}-T_{\mathrm{c}} \alpha_{P N}^{c}-\tau_{P N} \Delta T\right)^{2} \frac{\mathrm{s}}{1+r_{c}+s}}{\left(\alpha_{P N}^{h} T_{h}-\tau_{P N} \Delta T\right)\left(T_{\mathrm{h}} \alpha_{P N}^{h}-T_{\mathrm{c}} \alpha_{P N}^{c}-\tau_{P N} \Delta T\right)+\left(K_{P}^{*}+K_{N}^{*}\right) \Delta T\left(1+r_{c}+s\right) R_{P N}+\frac{\left(T_{\mathrm{h}} \alpha_{P N}^{h}-T_{\mathrm{c}} \alpha_{P N}^{c}-\tau_{P N} \Delta T\right)^{2}}{1+r_{c}+s}\left(\frac{R_{P}^{*}+R_{N}^{*}}{R_{P N}}-1-r_{c h}\right)}
$$

Defining the dimensionless parameters to simplify the above equations, 


$$
\frac{\alpha_{P N}^{h}}{\alpha_{P N}^{c}}=\mu, \frac{\tau_{P N}}{\alpha_{P N}^{\mathrm{c}}}=v, \frac{K_{P}^{*}+K_{N}^{*}}{K_{P}+K_{N}}=\omega, \frac{R_{P}^{*}+R_{N}^{*}}{R_{P N}}=\theta
$$

Then Eq. (22) can be rewritten as

$$
\eta=\frac{\left(T_{h} \mu-T_{\mathrm{c}}-\Delta T \nu\right)^{2} \frac{\mathrm{s}}{1+r_{c}+s}}{\left(T_{h} \mu-\Delta T \nu\right)\left(T_{h} \mu-T_{c}-\Delta T \nu\right)+\frac{1}{Z_{P \mathrm{~N}}^{c}} \omega \Delta T\left(1+r_{c}+s\right)+\frac{\left(T_{h} \mu-T_{c}-\Delta T \nu\right)^{2}}{1+r_{c}+s}\left(\theta-1-r_{c h}\right)}
$$

It is obvious that the efficiency of the TEG is determined by many factors. For the given TE materials, the $\mathrm{Z}$ value of the device is can be optimized by changing the cross-section area ratio. The larger the $\mathrm{Z}$ is, the higher efficiency will be. The maximum $\mathrm{Z}$ of the module is given by

$$
Z_{P N}^{\mathrm{c}}=\frac{\left(\alpha_{P N}^{c}\right)^{2}}{K_{P N} R_{P N}} \leq Z_{M A X}^{c}=\frac{\left(\alpha_{P N}^{c}\right)^{2}}{\left(\sqrt{\lambda_{N} \rho_{N}}+\sqrt{\lambda_{P} \rho_{P}}\right)^{2}}\left(\text { when, } A_{P}=A_{N} \frac{L_{P}}{L_{N}} \sqrt{\frac{\rho_{P} \lambda_{N}}{\rho_{N} \lambda_{P}}}\right)
$$

The efficiency of the module can be rearranged as follows

$$
\eta=\frac{\left(T_{\mathrm{h}} \mu-T_{\mathrm{c}}-\Delta T V\right)^{2}}{\frac{\left(T_{\mathrm{h}} \mu-T_{\mathrm{c}}-\Delta T V\right)\left[\left(T_{\mathrm{h}} \mu-\Delta T V\right)\left(\theta+r_{c c}\right)+T_{\mathrm{c}}\left(1+r_{c h}-\theta\right)\right]+\frac{1}{Z_{p V}^{\mathrm{c}}} \omega \Delta T\left(1+r_{c}\right)^{2}}{s}+\frac{1}{Z_{p V}^{\mathrm{c}}} \omega \Delta T \cdot s+\left[\frac{2}{Z_{p V}^{\mathrm{c}}} \omega \Delta T\left(1+r_{c}\right)+\left(T_{h} \mu-\Delta T v\right)\left(T_{\mathrm{h}} \mu-T_{\mathrm{c}}-\Delta T v\right)\right]}
$$

Known from Eq. (26), the maximum efficiency of the module can be achieved when the denominator reaches the peak value,

$$
\eta \leq \eta_{M A X}=\frac{\left(T_{\mathrm{h}} \mu-T_{\mathrm{c}}-\Delta T v\right)^{2}}{\sqrt[2]{\left.\frac{1}{Z_{\mathrm{c}}^{\mathrm{c}}} \omega \Delta T\left\{\left(T_{\mathrm{h}} \mu-T_{\mathrm{c}}-\Delta T v\right)\left(T_{h} \mu-\Delta T v\right)\left(\theta+r_{c c}\right)+T_{\mathrm{c}}\left(1+r_{c h}-\theta\right)\right]+\frac{1}{Z_{p v}^{\mathrm{c}}} \omega \Delta T\left(1+r_{c}\right)^{2}\right\}+\left[\frac{2}{Z_{c v}^{\mathrm{c}}} \omega \Delta T\left(1+r_{c}\right)+\left(T_{h} \mu-\Delta T v\right)\left(T_{\mathrm{h}} \mu-T_{\mathrm{c}}-\Delta T v\right)\right]}}
$$

when

$$
\mathrm{s}=\sqrt{\frac{\left(T_{\mathrm{h}} \mu-T_{\mathrm{c}}-\Delta T V\right)\left[\left(T_{h} \mu-\Delta T \nu\right)\left(\theta+r_{c c}\right)+T_{\mathrm{c}}\left(1+r_{c h}-\theta\right)\right]+\frac{1}{Z_{P N}^{\mathrm{c}}} \omega \Delta T\left(1+r_{c}\right)^{2}}{\frac{1}{Z_{P N}^{\mathrm{c}}} \omega \Delta T}}
$$

The power output of the module can further simplified to

$$
P=\left(T_{\mathrm{h}} \alpha_{P N}^{h}-T_{\mathrm{c}} \alpha_{P N}^{c}-\tau_{P N} \Delta T\right)^{2} \frac{\mathrm{s}}{\left(1+r_{c}+s\right)^{2} R_{P N}}
$$

Taking typical $\mathrm{Bi}_{2} \mathrm{Te}_{3}$-based $\mathrm{P}$, N-type thermos-pellets (HZ-2 module, Technology, Inc.) for example, when $\lambda_{P}=1.4 \mathrm{~W} / \mathrm{K}, \lambda_{N}=1.1 \mathrm{~W} / \mathrm{K}, L_{P}=L_{N}=0.298 \mathrm{~cm}, A_{P}=A_{N}=0.0225 \mathrm{~cm}^{2}, \tau_{P}=9.33 \times 10^{-5}$ $\mathrm{V} / \mathrm{K}, \tau_{N}=1.86 \times 10^{-5} \mathrm{~V} / \mathrm{K}, I=0.5 \mathrm{~A},\left|\frac{\tau_{\tau} I L_{P}}{\lambda_{p} A_{P}}\right|=0.0441<<1,\left|\frac{\tau_{N} I L_{N}}{\lambda_{N} A_{N}}\right|=0.0112<<1$. Then the $K_{P}^{*}$, $K_{N}^{*}, R_{P}^{*}, R_{P}^{*}$ can be approximated as

$$
K_{P}^{*} \cong K_{P}\left(1-\frac{\tau_{P} I L_{P}}{2 \lambda_{P} A_{P}}\right), K_{N}^{*} \cong K_{N}\left(1+\frac{\tau_{N} I L_{N}}{2 \lambda_{N} A_{N}}\right)
$$


$R_{P}^{*} \cong \frac{R_{P}}{2}\left(1+\frac{\tau_{P} I L_{P}}{6 \lambda_{P} A_{P}}\right), R_{N}^{*} \cong \frac{R_{N}}{2}\left(1-\frac{\tau_{N} I L_{N}}{6 \lambda_{N} A_{N}}\right)$,

Substitution of Eq. (30)-(31) into Eq. (13)-(14) yields,

$$
\begin{aligned}
& q_{h}=\alpha_{P N}^{h} T_{h} I+\left(K_{P N}-\frac{1}{2} \tau_{P N} I\right) \Delta T-\frac{1}{2} I^{2} R_{P N}+\frac{I^{3}}{12}\left(\tau_{P} \frac{R_{P}}{K_{P}}-\tau_{N} \frac{R_{N}}{K_{N}}\right)-\tau_{P N} \Delta T I-I^{2} R_{c h} \\
& q_{c}=\alpha_{P N}^{\mathrm{c}} T_{c} I+\left(K_{P N}-\frac{1}{2} \tau_{P N} I\right) \Delta T+\frac{1}{2} I^{2} R_{P N}+\frac{I^{3}}{12}\left(\tau_{P} \frac{R_{P}}{K_{P}}-\tau_{N} \frac{R_{N}}{K_{N}}\right)+I^{2} R_{\mathrm{cc}}
\end{aligned}
$$

Then the efficiency of the module can be simplified as follows

$$
\eta=\frac{P}{q_{h}}=\frac{\left(T_{\mathrm{h}} \alpha_{P N}^{h}-T_{\mathrm{c}} \alpha_{P N}^{c}\right) I-\tau_{P N} \Delta T I-I^{2} R_{P N}-I^{2} R_{\mathrm{c}}}{\alpha_{P N}^{h} T_{h} I+K_{P N} \Delta T-\frac{3}{2} \tau_{P N} \Delta T I-\frac{1}{2} I^{2} R_{P N}+\frac{I^{3}}{12}\left(\tau_{P} \frac{R_{P}}{K_{P}}-\tau_{N} \frac{R_{N}}{K_{N}}\right)-I^{2} R_{c h}}
$$

In some simplified models, the Seebeck coefficients of the P, N-type thermos-pellets are set to be constants. On doing this, the electrical current and efficiency of the module can be further simplified as follows

$$
\begin{aligned}
& I=\frac{\Delta T \alpha_{P N}}{R_{G}+R_{L}} \\
& q_{h}=\alpha_{P N} T_{h} I+K_{P N} \Delta T-\frac{1}{2} I^{2} R_{P N}-I^{2} R_{c h} \\
& \eta=\frac{P}{q_{h}}=\frac{s \Delta T}{T_{h}\left(1+r_{c}+s\right)+\frac{1}{Z_{P N}}\left(1+r_{c}+s\right)^{2}-\left(\frac{1}{2}+r_{c h}\right) \Delta T}
\end{aligned}
$$

where

$$
\begin{gathered}
Z_{P N}=\frac{\alpha_{P N}^{2}}{K_{P N} R_{P N}} \leq Z_{M A X}=\frac{\alpha_{P N}^{2}}{\left(\sqrt{\lambda_{N} \rho_{N}}+\sqrt{\lambda_{P} \rho_{P}}\right)^{2}}\left(\text { when, } A_{P}=A_{N} \frac{L_{P}}{L_{N}} \sqrt{\frac{\rho_{P} \lambda_{N}}{\rho_{N} \lambda_{P}}}\right) \\
\text { When } \frac{\partial \eta}{\partial s}=0, \quad s=\sqrt{\left(1+r_{c}\right)^{2}+\left[\left(\frac{1}{2}+r_{c c}\right) T_{h}+\left(\frac{1}{2}+r_{c h}\right) T_{c}\right] \cdot Z_{P N}} \\
\eta_{M A X}=\frac{\Delta T}{2 \sqrt{\frac{1}{Z_{P N}}\left[\left(\frac{1}{2}+r_{c c}\right) T_{h}+\left(\frac{1}{2}+r_{c h}\right) T_{c}+\frac{1}{Z_{P N}}\left(1+r_{c}\right)^{2}\right]}+\frac{2}{Z_{P N}}\left(1+r_{c}\right)+T_{h}}
\end{gathered}
$$

If the heat loss and thermal resistance on the cold and hot end are not considered, the maximum efficiency of the model can be further simplified to Eq. (1). It is obvious that the maximum efficiency of the model is determined by the temperature at the cold and hot ends, and ZT value of thermoelectric materials. This is exactly the one-dimensional ideal TEG model[3]. Though the one-dimensional model is simple and straightforward, there are some flaws. When the length of the TEG approaches zero, the model predicts an infinite power output of the system. However, it will never happen in real circumstance. Fortunately, the more accurate model we developed above can eliminate the flaw. 
To evaluate how much the electrical resistance and Thompson Effect may influence the performance of commercial modules[38], we use three mathematical models to analyze the commercial HZ-2 module. Here the value of electrical contact resistance is estimated from the device dimension level, and the Thompson coefficient is calculated from the property data sheet in the company website[38].

As shown in Figure 2(a), all the three models can predict the performance of the device with reasonable accuracy, however, the improved model (model III) has the best accuracy. The maximum power output of a couple of thermo-pellets is about $6.5 \times 10^{-3} \mathrm{~W}$, and the peak power output of the module is about $0.65 \mathrm{~W}$. It should be noted that the coarsest model (Model I) matches the experiment data even better than the improved Model II. This happens not because Model I is better, but rather because the deviations introduced by electrical resistance and Thompson Effect counteract with each other. In Figure 2(b), Model I best matches with the experiment data, while Model III slightly overestimates the efficiency and model two underestimates the performance. The coarse model has good performance here, because the Thompson heat here is coincidently in the same direction with Fourier heat flow, counteracting the contact layer Joule heat. Here, performance of Model III can be further improved by introducing more accurate thermoelectric material property expressions. In Figure 2(c), Model III can estimate the power output vs electrical current best. However, in the high current range, the model underestimates the performance of the module. If we want to achieve better accuracy, more precise Thompson coefficient consideration should be adopted in the calculation.

\section{TEG device-level performance}

In a real TEG system (Figure 1), heat sinks/exchangers are often fixed at the hot and cold ends to maximum the highest temperature difference between the thermo-pellets. To protect the thermoelectric materials from cracks and evaporation, some thermal and electrical insulation materials are introduced to fill the gaps between $\mathrm{P}, \mathrm{N}$-type thermo-pellets. In the following section, we will present a model to predict the device level thermal performance of TEG.

Supposing the thermal conductivity in the hot and cold end are $K_{H}$ and $K_{C}$, respectively, the total thermal conductance in the hot and cold end are given by

$\frac{1}{K_{H}}=\frac{1}{K_{C H}}+\frac{1}{K_{C C H}}+\frac{1}{K_{S H}}=\frac{1}{f_{h}} \frac{1}{K_{P N}}$

$\frac{1}{K_{C}}=\frac{1}{K_{C C}}+\frac{1}{K_{C C C}}+\frac{1}{K_{S C}}=\frac{1}{f_{c}} \frac{1}{K_{P N}}$

where $K_{C H}, K_{C C H}$ and $K_{S H}$ are thermal conductance of hot end ceramic cover, contact layer and heat sink, respectively. $K_{C C}, K_{C C C}$ and $K_{S C}$ are thermal conductance of cold end ceramic cover, contact layer and heat sink, respectively.

To accurately calculate the performance of the whole device, the thermal conductance through the gas filled space is given by

$K_{g}=\frac{\lambda_{g} A_{g}}{L_{\mathrm{g}}}+\varepsilon \sigma A_{g}\left(T_{h}^{2}+T_{c}^{2}\right)\left(T_{h}+T_{c}\right)=f_{g} K_{P N}$ 
where $\lambda_{g}, A_{g}, L_{\mathrm{g}}, \varepsilon$ are thermal conductivity of air, the cross-section area of the gas passage, gas passage length and emissivity of the ceramic plate surface. Since $T_{h}$ and $T_{c}$ are functions of hot and cold source temperature, $f_{g}$ will slightly fluctuate during the calculation. We assume it is constant. Here, $\sigma=5.67 \times 10^{-8} \mathrm{Wm}^{-2} \mathrm{~K}^{-4}$ is the Stefan-Boltzmann constant. In order to reduce the thermal stress and heat leakage in the modules, thermal isolation material is often employed filling the air gaps. For this situation, only the first term should be kept in Eq. (42).

Heat flow through the gas filled space can be expressed as

$q_{g}=K_{g}\left(T_{h}-T_{c}\right)$

The energy conservation equation in the hot and cold end can be described as follow

$q_{h}+q_{g}=K_{H}\left(T_{H}-T_{h}\right)$

$P=q_{h}-q_{c}$

Substituting Eq. (17), (19) and (34) into Eq. (44)-(45), the above equations can be rewritten as

$\left\{\begin{array}{l}K_{H}\left(T_{H}-T_{h}\right)=\alpha_{P N}^{h} T_{h} I+\left(K_{P N}+K_{g}\right) \Delta T-\frac{1}{2} I^{2} R_{P N}+\frac{I^{3}}{12}\left(\tau_{P} \frac{R_{P}}{K_{P}}-\tau_{N} \frac{R_{N}}{K_{N}}\right)-\frac{3}{2} \tau_{P N} \Delta T I-I^{2} R_{c h} \\ K_{H}\left(T_{H}-T_{h}\right)-K_{C}\left[\left(T_{h}-\Delta T\right)-T_{C}\right]=I^{2} R_{L}\end{array}\right.$

The electrical current flowing through the thermoelectric material is given by

$I=\frac{T_{h} \alpha_{P N}^{h}-T_{\mathrm{c}} \alpha_{P N}^{c}-\tau_{P N} \Delta T}{R_{G}+R_{L}}=\frac{\left(\alpha_{P N}^{c}-\tau_{P N}\right) \Delta T+T_{h}\left(\alpha_{P N}^{h}-\alpha_{P N}^{c}\right)}{R_{G}+R_{L}}$

Eq. (47) can be rearranged as

$I=\frac{T_{h} \alpha_{P N}^{h}-T_{\mathrm{c}} \alpha_{P N}^{c}-\tau_{P N} \Delta T}{R_{G}+R_{L}}=\frac{\left(\alpha_{P N}^{h}-\tau_{P N}\right) \Delta T+T_{\mathrm{c}}\left(\alpha_{P N}^{h}-\alpha_{P N}^{c}\right)}{R_{G}+R_{L}}$

Adding Eq. (47) into Eq. (48),

$I=\frac{\left(\frac{\alpha_{P N}^{h}+\alpha_{P N}^{c}}{2}-\tau_{P N}\right) \Delta T+\frac{T_{h}+T_{c}}{2}\left(\alpha_{P N}^{h}-\alpha_{P N}^{c}\right)}{R_{G}+R_{L}}$

If we assume the Thompson coefficient is constant for our model, then

$\frac{T_{h}+T_{c}}{2}\left(\alpha_{P N}^{h}-\alpha_{P N}^{c}\right) \cong \tau_{P N} \Delta T$

Eq. (51) can be further simplified to

$I=\frac{\alpha_{P N}^{h}+\alpha_{P N}^{c}}{2\left(R_{G}+R_{L}\right)} \Delta T$

Substituting Eq. (51) into the Eq. (46), we obtain

$C_{1} \cdot \Delta T^{3}+C_{2} \cdot \Delta T^{2}+C_{3} \cdot \Delta T+C_{4}=0$

where

$C_{1}=-\frac{s \alpha_{P N}^{h}\left(\alpha_{P N}^{h}+\alpha_{P N}^{c}\right)^{3}}{8\left(K_{H}+K_{C}\right)\left(1+r_{c c}+r_{c h}+s\right)^{3} R_{P N}^{2}}+\frac{1}{96} \frac{\left(\alpha_{P N}^{h}+\alpha_{P N}^{c}\right)^{3}}{\left(1+r_{c c}+r_{c h}+s\right)^{3} R_{P N}^{3}}\left(\tau_{P} \frac{R_{P}}{K_{P}}-\tau_{N} \frac{R_{N}}{K_{N}}\right)$ 


$$
\begin{aligned}
& C_{2}=\frac{K_{C} \alpha_{P N}^{h}\left(\alpha_{P N}^{h}+\alpha_{P N}^{c}\right)}{2\left(K_{H}+K_{C}\right)\left(1+r_{c c}+r_{c h}+s\right) R_{P N}}-\frac{K_{H}\left(\alpha_{P N}^{h}+\alpha_{P N}^{c}\right)^{2} s}{4\left(K_{H}+K_{C}\right)\left(1+r_{c c}+r_{c h}+s\right)^{2} R_{P N}}-\frac{\left(\alpha_{P N}^{h}+\alpha_{P N}^{c}\right)^{2}\left(1+2 r_{c h}\right)}{8\left(1+r_{c c}+r_{c h}+s\right)^{2} R_{P N}}-\frac{3 \tau_{P N}\left(\alpha_{P N}^{h}+\alpha_{P N}^{c}\right)}{4\left(1+r_{c c}+r_{c h}+s\right) R_{P N}} \\
& C_{3}=\frac{\left(K_{H} T_{H}+K_{C} T_{C}\right) \cdot \alpha_{P N}^{h}\left(\alpha_{P N}^{h}+\alpha_{P N}^{h}\right)}{2\left(K_{H}+K_{C}\right)\left(1+r_{c c}+r_{c h}+s\right) R_{P N}}+K_{P N}+K_{g}+\frac{K_{C} K_{H}}{K_{H}+K_{C}} \\
& C_{4}=-K_{H} T_{H}+\frac{\left(K_{H} T_{H}+K_{C} T_{C}\right) K_{H}}{K_{H}+K_{C}}
\end{aligned}
$$

This is a cubic equation that can be solved by the Cartan formula. The commercial HZ-2 TEG module fabricated by Hi-Z Technology, Inc., is employed to validate the model's accuracy. In the module, $\lambda_{P}=1.4 \mathrm{~W} / \mathrm{K}, \lambda_{N}=1.1 \mathrm{~W} / \mathrm{K}, L_{P}=L_{N}=2.98 \mathrm{~mm}, A_{P}=A_{N}=1.5 \times 1.5 \mathrm{~mm}^{2}, \rho_{\mathrm{P}}=1.3 \times 10^{-5} \Omega \cdot \mathrm{m}$, $\rho_{\mathrm{N}}=1.4 \times 10^{-5} \Omega \cdot m, \quad \alpha_{P}^{h}=2.1 \times 10^{-4} V / K, \quad \alpha_{P}^{c}=1.85 \times 10^{-4} \quad V / K, \quad \alpha_{N}^{c}=-1.80 \times 10^{-4} \quad V / K, \quad \alpha_{N}^{c}=-1.75 \times 10^{-4} V / K$, $\tau_{P}=9.33 \times 10^{-5} \mathrm{~V} / \mathrm{K}, \tau_{N}=1.86 \times 10^{-5} \mathrm{~V} / \mathrm{K}, I=0.5 \mathrm{~A}, K_{P}=1.06 \times 10^{-3} \mathrm{~W} \cdot \mathrm{m} / \mathrm{K}, K_{P}=0.83 \times 10^{-3} \mathrm{~W} \cdot \mathrm{m} / \mathrm{K}$, $R_{P}=1.72 \times 10^{-3} \Omega, R_{N}=1.85 \times 10^{-3} \Omega, f_{g}=f_{c}=1.0, r_{c c}=r_{c h}=0$. Substituting these values into the first and second terms of $\mathrm{C} 1$,

$$
\left\{\begin{array}{l}
\frac{s \alpha_{P N}^{h}\left(\alpha_{P N}^{h}+\alpha_{P N}^{c}\right)^{3}}{8\left(K_{H}+K_{C}\right)\left(1+r_{c c}+r_{c h}+s\right)^{3} R_{P N}^{2}}<\frac{\alpha_{P N}^{h}\left(\alpha_{P N}^{h}+\alpha_{P N}^{c}\right)^{3}}{8\left(K_{H}+K_{C}\right)\left(1+r_{c c}+r_{c h}\right)^{2} R_{P N}^{2}} \sim 10^{-10} \\
\frac{1}{96} \frac{\left(\alpha_{P N}^{h}+\alpha_{P N}^{c}\right)^{3}}{\left(1+r_{c c}+r_{c h}+s\right)^{3} R_{P N}^{3}}\left(\tau_{P} \frac{R_{P}}{K_{P}}-\tau_{N} \frac{R_{N}}{K_{N}}\right)<\frac{1}{96} \frac{\left(\alpha_{P N}^{h}+\alpha_{P N}^{c}\right)^{3}}{\left(1+r_{c c}+r_{c h}\right)^{3} R_{P N}^{3}}\left(\tau_{P} \frac{R_{P}}{K_{P}}-\tau_{N} \frac{R_{N}}{K_{N}}\right) \sim 10^{-11}
\end{array}\right.
$$

For the HZ-2 TEG module, $\Delta T$ is less than $200 K$ for most situations, so the value of the first term of the equation is in order of $10^{-3}$. The values of other terms are of magnitude 10 . When solving the equation, the first term can be neglected with little derivation, reducing the equation to a quadratic. Solving the coupled equations above, then we obtain

$$
\left\{\begin{array}{l}
T_{h}=f\left(T_{H}, T_{C}\right) \\
I=g\left(T_{H}, T_{C}\right) \\
T_{c}=h\left(T_{H}, T_{C}\right)
\end{array}\right.
$$

In the case when $\alpha_{P N}^{h}=\alpha_{P N}^{\mathrm{c}}=\alpha_{P N}=\alpha, \tau_{P N}=0$, the above equations can further be simplified as follows

$$
\left\{\begin{array}{l}
K_{H}\left(T_{H}-T_{h}\right)=\frac{\alpha^{2} \cdot \Delta T \cdot T_{h}}{R_{G}+R_{L}}+K_{P N} \cdot \Delta T-\frac{1}{2}\left(\frac{\alpha \Delta T}{R_{G}+R_{L}}\right)^{2}\left(R_{P N}+2 R_{c h}\right) \\
K_{H}\left(T_{H}-T_{h}\right)-K_{C}\left[\left(T_{h}-\Delta T\right)-T_{C}\right]=\left(\frac{\alpha \Delta T}{R_{G}+R_{L}}\right)^{2} R_{L}
\end{array}\right.
$$

The factors in the cubic equation will reduced to

$$
\begin{aligned}
& C_{1}=\frac{-s \alpha_{P N}^{4}}{\left(K_{H}+K_{C}\right)\left(1+r_{c c}+r_{c h}+s\right)^{3} R_{P N}^{2}} \sim-10^{-10} \\
& C_{2}=\frac{K_{C} \cdot \alpha_{P N}^{2}}{\left(K_{H}+K_{C}\right)\left(1+r_{c c}+r_{c h}+s\right) R_{P N}}-\frac{K_{H} \cdot \alpha_{P N}^{2} \cdot s}{\left(K_{H}+K_{C}\right)\left(1+r_{c c}+r_{c h}+s\right)^{2} R_{P N}}-\frac{\alpha_{P N}^{2}(1+2 \cdot r c h)}{2\left(1+r_{c c}+r_{c h}+s\right)^{2} R_{P N}} \\
& C_{3}=\frac{\left(K_{H} T_{H}+K_{C} T_{C}\right) \cdot \alpha_{P N}^{2}}{\left(K_{H}+K_{C}\right)\left(1+r_{c c}+r_{c h}+s\right) R_{P N}}+K_{P N}+K_{g}+\frac{K_{C} K_{H}}{K_{H}+K_{C}} \\
& C_{4}=-K_{H} T_{H}+\frac{\left(K_{H} T_{H}+K_{C} T_{C}\right) K_{H}}{K_{H}+K_{C}}
\end{aligned}
$$

The value of the first term of the equation is of magnitude $10^{-3}$. The values of other terms are 
of magnitude 10, so the first term can be neglected with little derivation when solving the equation. Eq. (58) is widely used to obtain the effective temperature drop in the thermos-pellets.

Comparing the calculation results of the three models on the commercial HZ-2 TEG module, we can evaluate the sensitivity of the TEG module to electrical resistance at the contact layer, filled gap heat flow leakage, and Thompson coefficients. In the coarsest model, the Thompson effect and contact layer electrical resistance are unaccounted for. The constant Thompson coefficient, the filled gap heat flow, and various contact layer electrical resistance are then added into the consideration for the improved models. The situations we employed are list in Table 1.

As Figure 3(a) shown, as the load resistance increases, the temperature difference between the hot and cold ends increases because lower load resistance means larger Peltier heat absorption at the ends. When the Thompson Effect is accounted for, when the contact layer electrical resistance is the same, the temperature difference is slightly lower than the coarse model (model one). In addition, the contact layer electrical resistance will significantly change the temperature difference. It can be reasons as larger contact electrical resistance leads to smaller electrical current, consequently less Peltier heat absorption at the two ends.

Figure 3(b) shows the electrical current output of the TEG device. It is obvious that the larger contact layer electrical resistance, the lower current output is. However, when the contact resistance has the same value, loading it on the hot or cold end brings some temperature distribution in the thermo-pins, but it has little impact on the current output of the system. In addition, the Thompson coefficient in the HZ-2 model slightly increases the current output. Figure 3(c) shows the power output of a couple of thermo-pellets in the device. It can be seen that, larger electrical resistance in the hot/cold end contact layer lowers the power output of the system. When the electrical contact layer is as large as the internal electrical resistance, the power output can be only half of that when no contact layer electrical resistance is considered. The Thompson effect does affect the power output of the system, with about $10 \%$ percent increase for the same electrical load resistance factor. In addition, the optimized electrical load resistance for the peak power output shifts to higher value. It is reasonable since the peak power output is achieved when load electrical resistance equals to internal resistance. It's safe to conclude that Thompson Effect cannot be neglected for accurate modeling analysis when temperature gradient is large $\left(\sim 5.0 \times 10^{4}\right.$ $\mathrm{K} / \mathrm{m}$ ) in the thermo-pellets, and that contact layer electrical resistance will largely reduce the maximum power output of the system.

As seen in Figure 3(d), the efficiency of the device increases quickly and then decreases gradually as the load electrical resistance increases. Here the efficiency of the improved model is slightly lower than coarse one. It can be reasoned as a combined result of two phenomena: on one hand, heat leakage through the thermal isolation material taken into consideration in the improved model reduces the module efficiency; on the other hand, Thompson Effect slightly increases the conversion efficiency. It reminds us that, through careful thermoelectric material doping, making the Seebeck coefficients gradient matched with the temperature gradient, we can maximize the current, power output, and efficiency of the device by taking the advantage of Thompson Effect. Moreover, the electrical resistance at the contact layer significantly lowers the efficiency of the whole system. Perfect contact layer is of vital importance for the device fabrication, especially when the device is micrometers thick. When contact layer electrical resistance increases, the electrical load resistance corresponding to peak efficiency shifts from low value to high value, and the efficiency of the device becomes un-sensitive to the load resistance. In MEMS fabrication, the 
device may have larger individual differences, because the contact layer resistance is difficult to control.

\section{Conclusion}

In this paper, we developed a more accurate model to evaluate the performance of a TEG module with the contact layer resistance, Thompson Effect, Joule heat, and thermo-pellet gap heat leakage all taking account for. The sensitivity of the factors, including hot and cold end electrical resistance, and Thompson Effect, which may affect the module's performance were analyzed. Through the modelling and analysis, we draw the following conclusions:

(1) The thermoelectric effect of Seebeck, Peltier, and Thomson effects are integrated with Fourier heat and Joule source terms to analyze the performance of the TEG modules. A constant Thompson coefficient is accounted and both full and simplified expressions of the voltage, current, power output, and efficiency are given by introducing some dimensionless parameters. Comparisons between the three models and experimental results validate the accuracy of the improved model. The results indicate that the Thompson Effect can become significant when the temperature gradient is relatively large in the module. Neglecting the effect can lead to as more as $10 \%$ derivation from the actual efficiency in some cases. Careful material doping can help to increase the efficiency of the device by taking advantage of Thompson Effect.

(2) Thermal and electrical resistance at the contact layers will no doubt deteriorate the performance of the TEG module. The contact layer thermal resistance reduces the effective temperature drop in the thermo-pellets, while the electrical resistance will largely lower the peak efficiency and power output of the device. The effect can be more notable for MEMS TEGs, because the internal resistances of such TEGs are small and tiny flaws in the contact layer will markedly deteriorate its performance.

(3) Thermal resistance of the ceramic covers and heat sinks/exchangers fixed at the two ends of TEG system is a factor decreasing the system performance. Heat leakage via the gap between the thermo-pellets is another factor reducing its efficiency. The narrower gap and filling materials with higher thermal resistivity are required for high performance TEG system design.

In this paper, the Thompson Effect is set to be a constant in order to linear the heat transfer control equation in the model. The improved model is more accurate and it is adaptable to analyze various TEG systems by specifying the geometry sizes and material properties of a given system. A further study using numerical tool to simulate the coupled effects of thermal-electric in the TEG system will be conducted in the near future, in which more sophisticated material properties expressions and better boundary control can be used to further reduce the derivations.

\section{Acknowledgement}

The authors wish to thank the support of US National Science Foundation via Grant \#1529842.

\section{Reference}

[1] Goldsmid HJ. Introduction to Thermoelectricity. Springer Ser Mater S. 2010;121:1-242. 
[2] Goldsmid HJ, Douglas RW. The Use of Semiconductors in Thermoelectric Refrigeration. Brit J Appl Phys. 1954;5(Nov):386-90.

[3] Rowe DM. Thermoelectrics handbook : macro to nano. Boca Raton: CRC/Taylor \& Francis, 2006.

[4] El-Genk MS, Saber HH, Caillat T. Efficient segmented thermoelectric unicouples for space power applications. Energ Convers Manage. 2003;44(11):1755-72.

[5] Basti A, Obikawa T, Shinozuka J. Tools with built-in thin film thermocouple sensors for monitoring cutting temperature. Int J Mach Tool Manu. 2007;47(5):793-8.

[6] Samson D, Kluge M, Fuss T, Schmid U, Becker T. Flight Test Results of a Thermoelectric Energy Harvester for Aircraft. J Electron Mater. 2012;41(6):1134-7.

[7] Roundy S, Steingart D, Frechette L, Wright P, Rabaey J. Power sources for wireless sensor networks. Lect Notes Comput Sc. 2004;2920:1-17.

[8] Longtin JP, Zuo L, Hwang D, Fu GS, Tewolde M, Chen YK, et al. Fabrication of Thermoelectric Devices Using Thermal Spray: Application to Vehicle Exhaust Systems. J Therm Spray Techn. 2013;22(5):577-87.

[9] Hicks LD, Dresselhaus MS. Thermoelectric Figure of Merit of a One-Dimensional Conductor. Phys Rev B. 1993;47(24):16631-4.

[10] Tian ZT, Lee S, Chen G. Heat Transfer in Thermoelectric Materials and Devices. J Heat Trans-T Asme. 2013;135(6).

[11] Harman TC, Walsh MP, Laforge BE, Turner GW. Nanostructured thermoelectric materials. J Electron Mater. 2005;34(5):L19-L22.

[12] Venkatasubramanian R, Siivola E, Colpitts T, O'Quinn B. Thin-film thermoelectric devices with high room-temperature figures of merit. Nature. 2001;413(6856):597-602.

[13] Mahan GD, Sofo JO. The best thermoelectric. P Natl Acad Sci USA. 1996;93(15):7436-9.

[14] Vining CB. An inconvenient truth about thermoelectrics. Nat Mater. 2009;8(2):83-5.

[15] Hsiao YY, Chang WC, Chen SL. A mathematic model of thermoelectric module with applications on waste heat recovery from automobile engine. Energy. 2010;35(3):1447-54.

[16] Wu C. Analysis of waste-heat thermoelectric power generators. Appl Therm Eng. 1996;16(1):63-9.

[17] Yu C, Chau KT. Thermoelectric automotive waste heat energy recovery using maximum power point tracking. Energ Convers Manage. 2009;50(6):1506-12.

[18] Clayton D, Willems R. Communication Requirements and Concept of Operation for Sensor Networks. Oak Ridge National Laboratory (ORNL); 2013.

[19] Lampinen MJ. Thermodynamic Analysis of Thermoelectric Generator. J Appl Phys. 1991;69(8):4318-23.

[20] Chen JC, Yan ZJ, Wu LQ. The influence of Thomson effect on the maximum power output and maximum efficiency of a thermoelectric generator. J Appl Phys. 1996;79(11):8823-8.

[21] Omer SA. Solar Thermoelectric System for Small Scale Power Generation [Doctoral Thesis]: Loughborough University 1997.

[22] Omer SA, Infield DG. Design optimization of thermoelectric devices for solar power generation. Sol Energ Mat Sol C. 1998;53(1-2):67-82.

[23] Freunek M, Muller M, Ungan T, Walker W, Reindl LM. New Physical Model for Thermoelectric Generators. J Electron Mater. 2009;38(7):1214-20.

[24] Rowe DM. Thermoelectric Handbook-Macro to Nano: Taylor \& Francis Group, 2006.

[25] Min G, Rowe DM. Improved model for calculating the coefficient of performance of a Peltier module. Energ Convers Manage. 2000;41(2):163-71. 
[26] Huang MJ, Yen RH, Wang AB. The influence of the Thomson effect on the performance of a thermoelectric cooler. Int J Heat Mass Tran. 2005;48(2):413-8.

[27] Snyder GJ, Lim JR, Huang CK, Fleurial JP. Thermoelectric microdevice fabricated by a MEMS-like electrochemical process. Nat Mater. 2003;2(8):528-31.

[28] Lenggenhager R, Baltes H, Peer J, Forster M. Thermoelectric Infrared-Sensors by Cmos Technology. Ieee Electr Device L. 1992;13(9):454-6.

[29] Chowdhury I, Prasher R, Lofgreen K, Chrysler G, Narasimhan S, Mahajan R, et al. On-chip cooling by superlattice-based thin-film thermoelectrics. Nat Nanotechnol. 2009;4(4):235-8.

[30] Sampath S. Thermal Spray Applications in Electronics and Sensors: Past, Present, and Future. J Therm Spray Techn. 2010;19(5):921-49.

[31] Noro H, Sato K, Kagechika H. The Thermoelectric Properties and Crystallography of Bi-Sb-Te-Se Thin-Films Grown by Ion-Beam Sputtering. J Appl Phys. 1993;73(3):1252-60.

[32] Leonov V, Torfs T, Fiorini P, Van Hoof C. Thermoelectric converters of numan warmth for self-powered wireless sensor nodes. Ieee Sens J. 2007;7(5-6):650-7.

[33] Francioso L, De Pascali C, Farella I, Martucci C, Creti P, Siciliano P, et al. Flexible thermoelectric generator for ambient assisted living wearable biometric sensors. J Power Sources. 2011;196(6):3239-43.

[34] IGshl M, Nemoto H, Hamao T, Yamimoto M, Sudou S, hdai M, et al. Micro thermoelectric modules and their application to wristwatches as an energy source. 18th International Conference on Thermoelectrics: IEEE; 1999.

[35] Stark I, Stordeur M. New Micro Thermoelectric Devices Based On Bismuth Telluride-Type Thin Solid Films. 18th International Conference on Thermoelectrics: IEEE; 1999.

[36] Huesgen T, Woias P, Kockmann N. Design and fabrication of MEMS thermoelectric generators with high temperature efficiency. Sensor Actuat a-Phys. 2008;145:423-9.

[37] Yan D. Modeling and Application of a Thermoelectric Generator: University of Toronto, 2011.

[38] Hi-Z Technology I. HZ-2 Thermoelectric Module. 2014. 


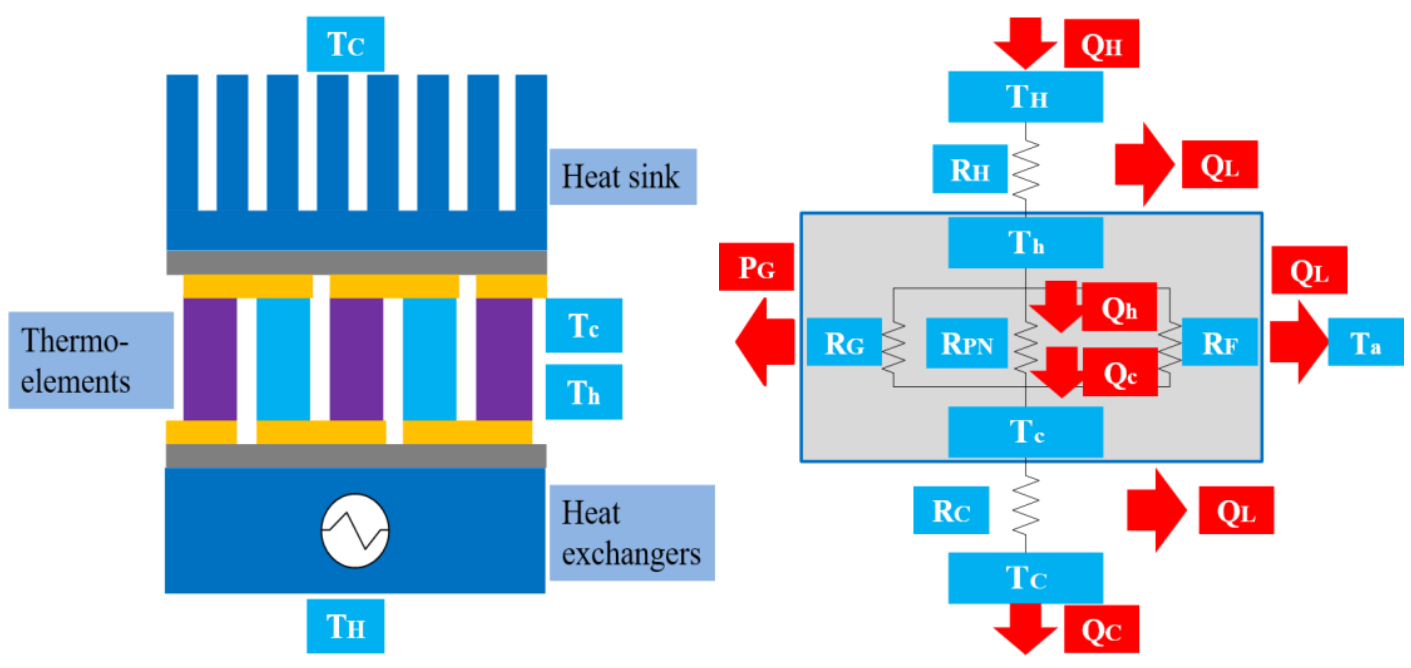

Figure 1 Schematic diagram of thermoelectric generator: (a) TEG model; (b) Thermal network

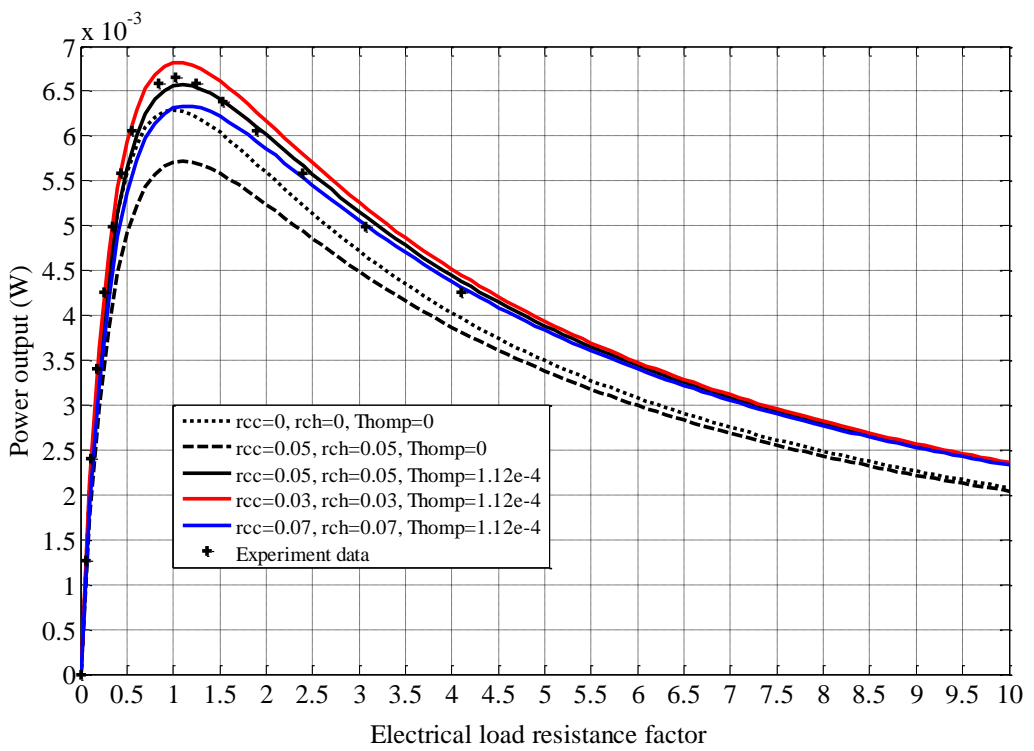

(a) 


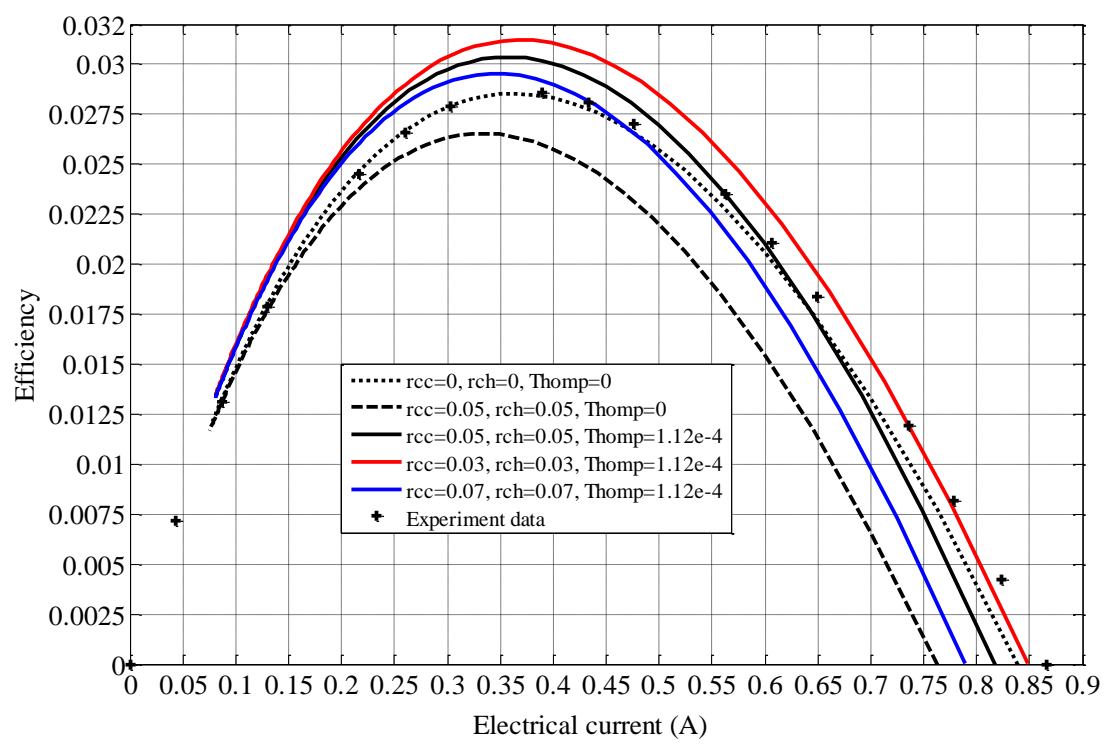

(b)

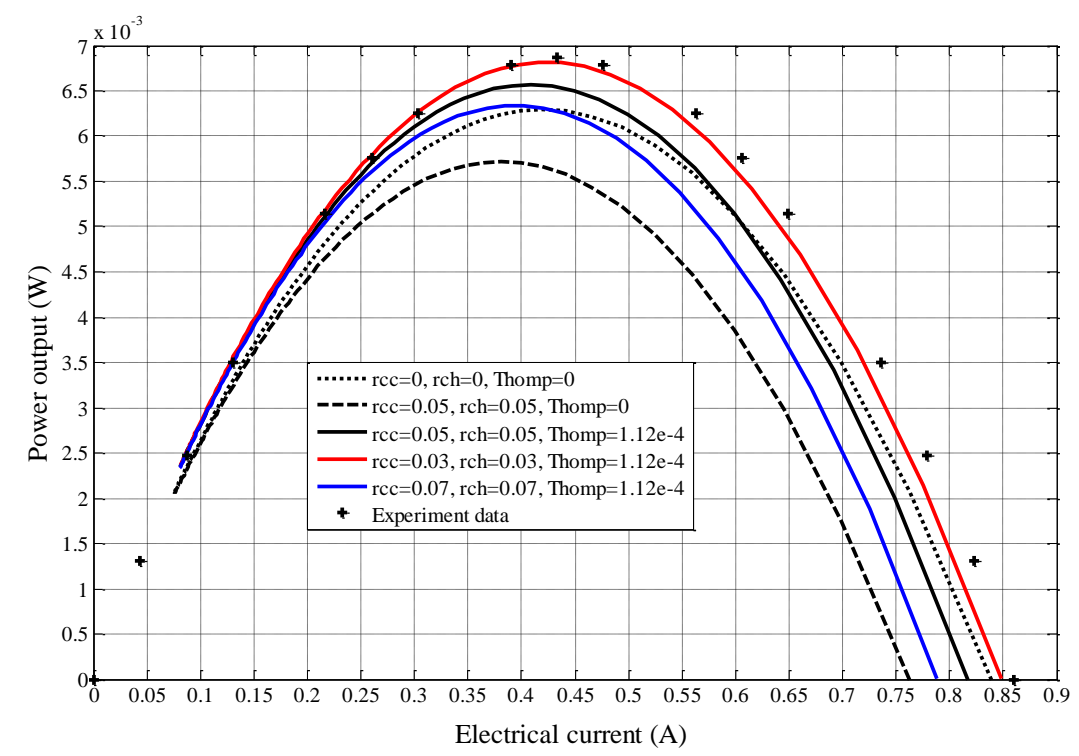

(c)

Figure 2 The performance modeling of a couple of thermo-pellets: (a) Power output of device vs load resistance; (b) Efficiency of device vs electrical current; (c) Power output vs electrical current. Model I: $r_{c c}=r_{c h}=0$ and $\tau_{P}=\tau_{N}=0$; Model II: $r_{c c}=r_{c h}=0.05$ and $\tau_{P}=\tau_{N}=0$; Model III: $r_{c c}=r_{c h}=0.05 / 0.03 / 0.07$ and $\tau_{P}=9.33 \times 10^{-5} \mathrm{~V} / \mathrm{K}, \tau_{N}=-1.86 \times 10^{-5} \mathrm{~V} / \mathrm{K} ; T_{h}=150{ }^{\circ} \mathrm{C}, T_{c}=50{ }^{\circ} \mathrm{C}$. 


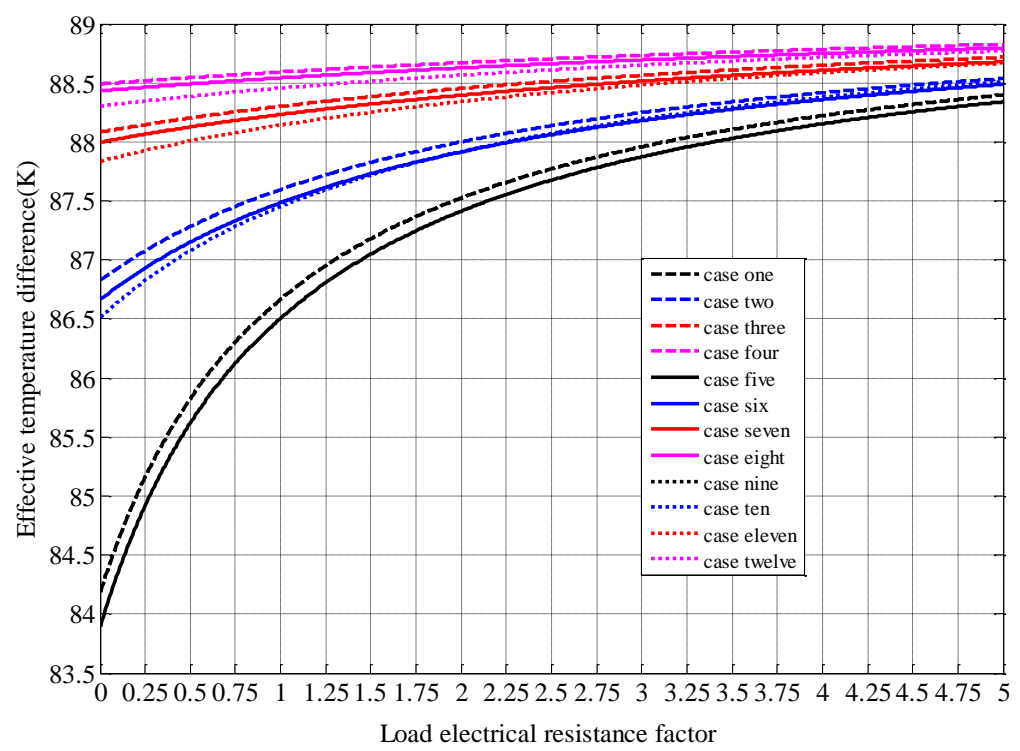

(a)

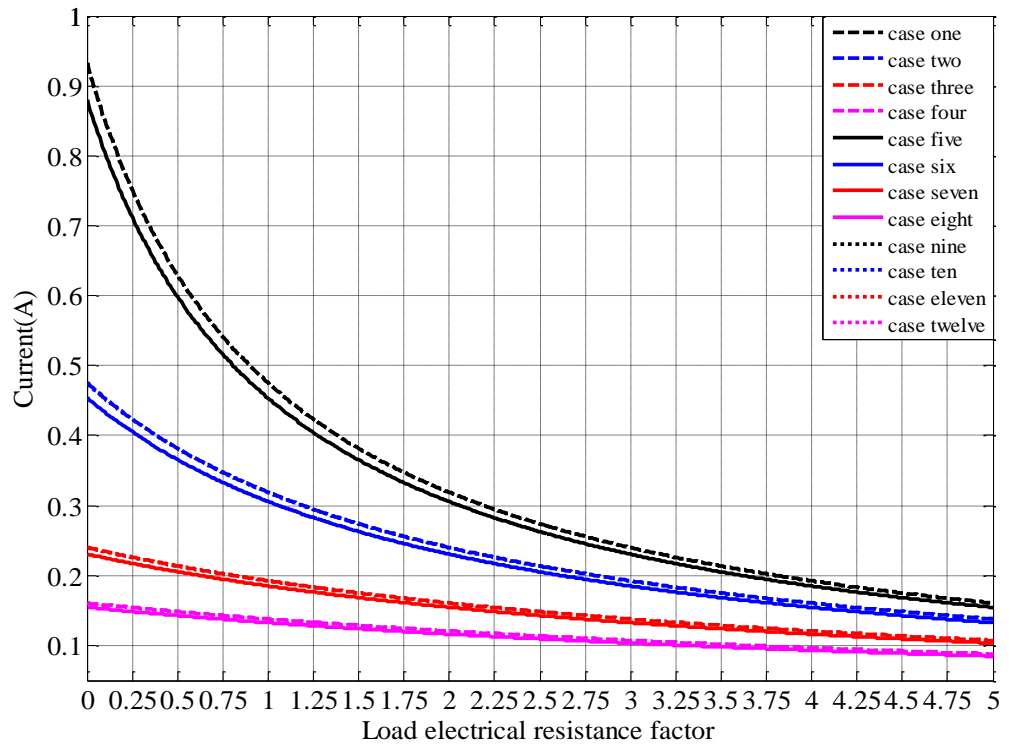

(b)

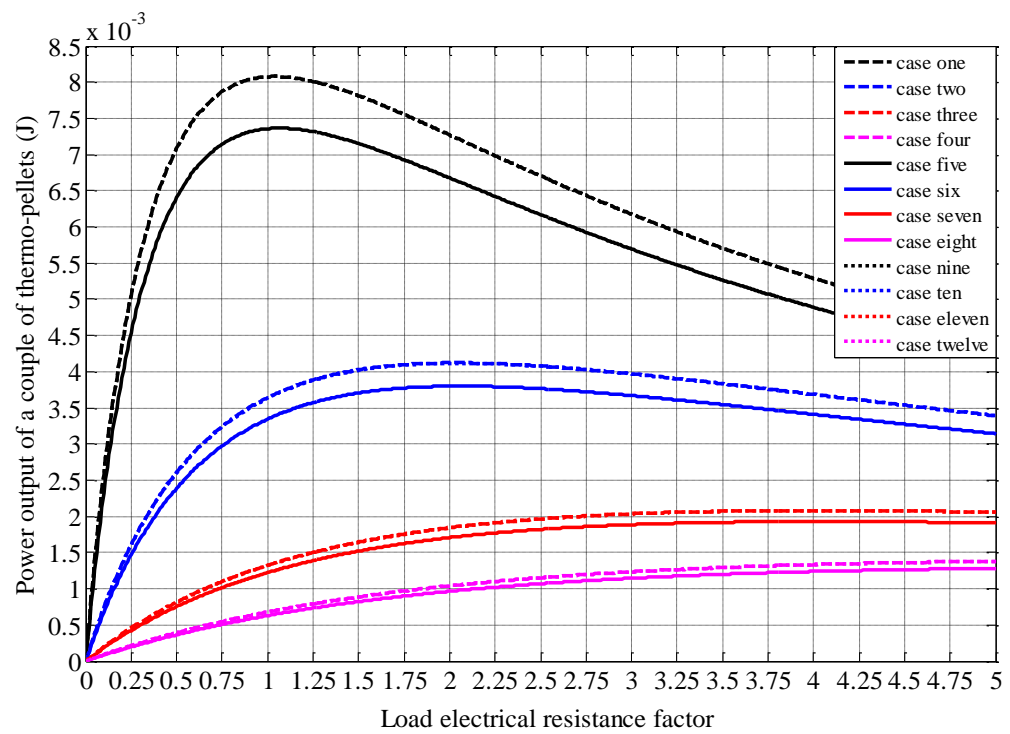


(c)

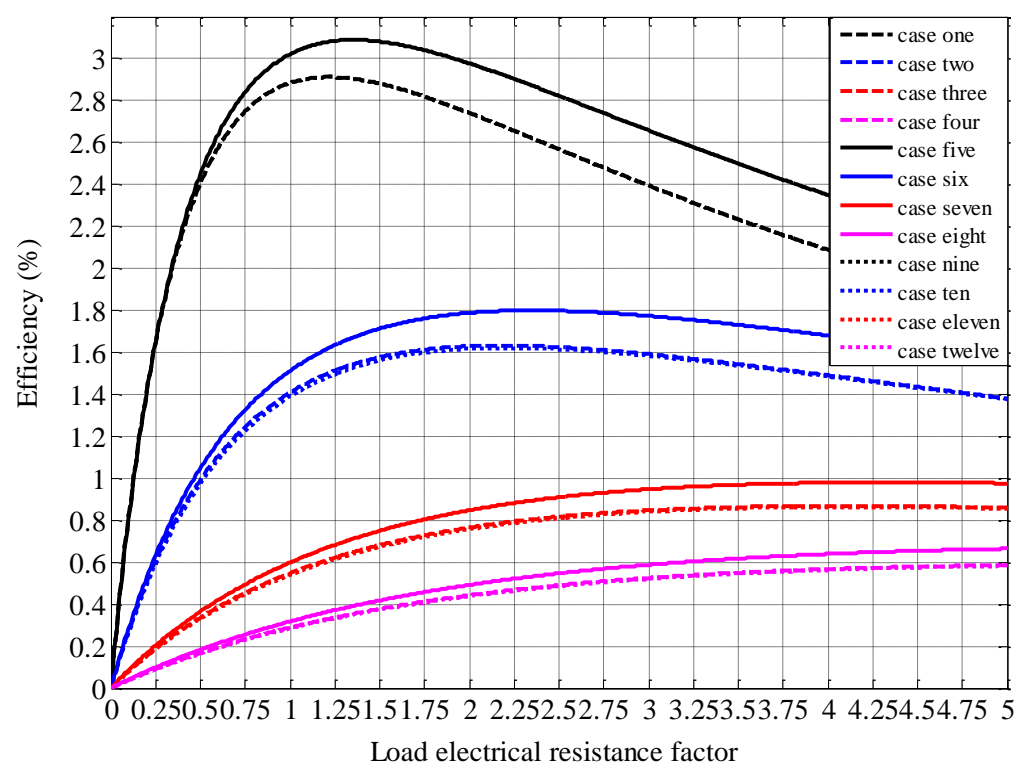

(d)

Figure 3 Modeling of TEG system based on commercial HZ-2 TEG module: (a) Effective temperature varies with load electrical resistance factor; (b) Current varies with load electrical resistance factor; (c) Power varies with load electrical resistance factor; (d) Efficiency varies with load electrical resistance factor.

Table 1. Cases to exam the models

\begin{tabular}{llll}
\hline \hline Cases & $\begin{array}{l}\text { Hot end contact electrical } \\
\text { resistance factor }\left(r_{c h}\right)\end{array}$ & $\begin{array}{l}\text { Hot end contact electrical } \\
\text { resistance factor }\left(r_{c c}\right)\end{array}$ & $\begin{array}{l}\text { Thompson } \\
\text { Coefficient }\left(\tau_{P N}\right)\end{array}$ \\
\hline Case one & 0 & 0 & $1.12 \times 10^{-4}$ \\
Case two & 1 & 0 & $1.12 \times 10^{-4}$ \\
Case three & 3 & 0 & $1.12 \times 10^{-4}$ \\
Case four & 5 & 0 & $1.12 \times 10^{-4}$ \\
\hline Case five & 0 & 0 & 0 \\
Case six & 1 & 0 & 0 \\
Case seven & 3 & 0 & 0 \\
Case eight & 5 & 0 & 0 \\
\hline Case nine & 0 & 0 & $1.12 \times 10^{-4}$ \\
Case ten & 0 & 1 & $1.12 \times 10^{-4}$ \\
Case eleven & 0 & 3 & $1.12 \times 10^{-4}$ \\
Case twelve & 0 & 5 & $1.12 \times 10^{-4}$ \\
\hline \hline
\end{tabular}

*Parameter values adopted in the modeling: $T_{H}=423 \mathrm{~K}, T_{C}=323 \mathrm{~K}, f_{H}=f_{C}=10, f_{g}=0.1$. 